\title{
¿EXISTE UNA PLAZA "LATINOAMERICANA" PARA ARBITRAR INTERNACIONALMENTE?
}

\author{
Fernando Cantuarias S.*
}

\begin{abstract}
Desde la década pasada, el mundo es testigo de como el arbitraje $^{1}$ ha dejado de ser un tema privativo de algunos pocos Estados, gracias a que muchos países, incluidos aquellos tradicionalmente considerados hostiles hacia el arbitraje, están adoptando modernas legislaciones y se adhieren a importantes tratados sobre la materia.
\end{abstract}

* Decano de la Facultad de Derecho de la Universidad Peruana de Ciencias Aplicadas (UPC). Abogado por la Pontificia Universidad Católica del Peń y Master en Derecho (LL.M.) por la Universidad de Yale. Árbitro nacional e intemacional. En el año 2006 ha sido reconocido por la prestigiosa revista Latinlawyer como uno de los 34 principales árbitros de América Latina. Además, la teputada revista Who's Who Legal -Commercial Arbitration 2007, Commercial Arbitration 2008 y Commercial Arbitration 2009- lo considera entre los 467 expertos lideres en este campo en 63 jurisdicciones. Es miembro del Board of Reporters del Institute of Transnational Arbitration (ITA), del Grupo Latinoamericano de Arbitraje de la Cámara de Comercio Internacional (CCI), del Comité Editor de la Revista Peruana de Arbitraje y del Comité Argentino de Arbitraje Nacional y Transnacional. Es miembro de la lista de árbitros de los principales Centros de Arbitraje del Pení y del Energy Arbitrators" list (ICDR). Es autor del Libro "Arbitraje Comercial y de las Inversiones" (UPC, Lima, 2007) y de más de 50 artículos sobre su especialidad.

I. En este artículo no analizaremos el arbitraje entre Estados, como tampoco el arbittaje entre Estados e inversionistas, que, como sabemos, también viene alcanzando un desarrollo impresionante, gracias principalmente a la Convención sobre Arreglo de Diferencias Relativas a Inversiones entre Estados y Nacionales de otros Estados (CIADI) y a los Tratados Bilaterales de Promoción y Protección Reciptoca de Inversiones (BITs). Sobre este particular, leer a: Fernando Cantuarias Salaverry, "Arbitraje Comercial y de las Inversiones", UPC, Lima, 2007, pp. 555-809; Femando Cantuarias Salaverry, "Condiciones para acceder al CIADI: A propósito del Caso Lucchetei". En: Revista de Economia y Derecho, vol. 1, nro. I, Universidad Peruana de Ciencias Aplicadas (UPC), Lima, 2004; Femando Cantuarias Salaverry, "Los Tratados Bilaterales de Promoción y Protección Reciproca de Inversiones y el acceso al Arbitraje". En: Revista de Economia y Derecho, vol. 1, nro. 2, Universidad Peruana de Ciencias Aplicadas (UPC), Lima, 2004, pp. 29-50; y, Fernando Cantuarias Salaverry, "La utilización de la vía arbitral en la solución de conflictos entre el Estado peruano y los inversionistas". En: Themis, Revista de Derecho, nro. 48, Lima, 2004, pp. 167-186. 
Carbonneau ${ }^{2}$ entiende que parte del mérito por el impresionante desarrollo del arbitraje se debe a la Ley Modelo de UNCITRAL sobre Arbitraje Comercial Internacional ${ }^{3}$, que ha sido adoptada, en todo o en parte, por más de cincuenta países alrededor del mundo: desde varios Estados latinoamericanos hasta Alemania, como por parte de Rusia y diversas jurisdicciones asiáticas. ${ }^{4}$

Otra parte del mérito lo tiene, qué duda cabe, el "Convenio sobre Reconocimiento y Ejecución de las Sentencias Arbitrales Extranjeras (más conocido como la Convención de Nueva York de 1958$)^{5}$, del que a abril de 2008 forman parte 142 Estados. ${ }^{6}$

2. Thomas E. Carbonneau, "The Ballad of Transborder Arbitration". En: University of Miami Law Review, vol. 56, 2002, p. 779. "During the 1990s, there was an eruption of Uncitral-inspired arbitra" tion laws throughout the globe -from Latin American countries to Germany to former Soviet bloc states and Asian jurisdictions".

3. La Ley Modelo de UNCITRAL no es un tratado, sino simplemente un modelo -valga la redundanciaque puede ser implementado por la legislación de cada país, con la finalidad de modemizar y uniformizar el tratamiento de los arbitrajes internacionales. Sobre el particular, leer a: Femando Cantuarias Salaverry, "Arbitraje Comercial y de las Inversiones", ob. cit., pp. 65-78; Gerold Hermann, "UNCITRAL's work towards a Model Law on International Commetcial Arbitration". En: Pace Law Review, vol. 4, nro. 3, 1984, pp. 547 y ss:; Gerold HerTmann, "The UNCITRAL Model Law its background, salient features and purposes". En: International Arbitration, vol. 1, nro. 1, 1989, pp. 13 y ss.; W. Laurence Craig, "Trends and Developments in the Laws and Practice of International Commercial Arbitration". En: Coudert Brothers Worldwide: http:/www.coudert.com/practice/intcom.htm; Alan Redfern \& Martin Hunter, "Law and Practice of International Commencial Arbitration", 2da. Ed., Sweet \& Maxwell, London, 1991, pp. 525 y ss.; y, Kenneth Ungar, "The Enforcement of Arbittal Awards under UNCITRAL's Model Law on International Commercial Arbitration". En: Columbia Journal of Transnational Law, vol. 25, nro. 3, 1987, pp. 727-74l.

4. Se han promulgado leyes basadas en la Ley Modelo de UNCITRAL sobre Arbjtraje Comercial Internacional en Alemania, Australia, Austria, Azerbaiyán, Bahrein, Bangladesh, Belarús, Bermudas, Bulgaria, Camboya, Canadá, Chile, Chipre, Croacia, Dinamarca, Egjpto, dentro del Rejno Unido de Gran Bretanta e Irlanda del Norte: Escocia, España, Estonia, Federación de Rusia, Filipinas, Grecia, Guatemala, Hong Kong (Regiơn administrativa especial de China), Hungría, India, Irán (República Islámica del), Irlanda, Japón, Jordania, Kenya, Lituania, Macao (Región administrativa especial de China), Madagascar, Malta, México, Niçaragua, Nigeria, Noruega, Nueva Zelandia, Omán, Paraguay, Perú, Polonia, República de Corea, Singapur, Sti Lanka, Tailandia, Túnez, Turquía, Ucrania, Uganda; dentro de los Estados Unidos de América: Califorria, Connecticut, Illinois, Louisiana, Oregón y Texas; Zambia y Zimbabwe. Sobre el particular, leer a: Bette E. Shifman, "Developments in adoption of the 1985 UNCITRAL Model Law on International Commercial Arbitration". En: The American Review of International Arbitration, wol. 1, nro. 2, 1990, pp. 281 y ss; Frank Griffith Dawson, "El rol del Poder Judicial en el proceso de arbitraje: ¿asistencia o intervención?". En: Fus Et Veritas, Revista de Derecho, nro. 15, Lima, 1997, p. 200; Gerold Hermann, "Power of Arbitrators to Determine Procedures under the UNCITRAL Model Law". En: ICCA XII International Arbitration Congress, Viena, 1994, p. 21; e, ICC Commission on Intemational Arbitration, "Final Report on Intellectual Property Disputes and Arbitration". En: The ICC International Court of Arbitration Bulletin, vol. 9, nro. 1, 1998, p. 47.

5. Richard J. Graving, "How Non-Contracting States to the "Universal' New York Arbitration Convention enjoy Third-Party Benefits but not Third-Party Rights". En; Journal of International

314 Revista Ecuatoriana de ARBITRAFE 
Pero, además, el éxito del arbitraje se debe a sí mismo, ya que se trata de un instrumento de inapreciable valor en la solución de los conflictos; en especial, de los que se generan del intercambio comercial internacional.

\author{
En efecto, si bien en el ámbito de los conflictos domésticos el \\ arbitraje viene demostrando una importancia cada vez mayor \\ frente al Poder Judicial ${ }^{7}$, es en la solución de los conflictos \\ comerciales internacionales donde destaca nítidamente, debido \\ a que, además:
}

Arbitration, vol. I4, nro. 3, 1997, p. 167. "The New York Arbitration Convention of 1958 is mercifully short and, for the international commencial community, successfully sweet. Without evident hyperbole Lord Mustill has called it 'perhaps the most effective instance of international legislation in the entire history of commercial law'. Or as President Stephen Schwebel of the International Court of Justice has put it with greater economy but no less accuracy, 'it works'. Yet another authority, Professor Thomas Carbonnealt, has described it as the 'universal charter' of international commercial arbitration". Sobre este particular, leer at Fernando Cantuarias Salaverry, "Arbitraje Comercial y de las Inversiones", ob. cit., pp. 42-62; Albert Jan van den Berg, "Non-domestic Arbitral Awards under the 1958 New York Convention". En: Arbitration Intemational, vol. 2, nro. 3, 1986, pp. 198 y ss.; Albert Jan van den Berg, "New York Convention of 1958: Consolidated Commentary, Cases reported in Volumes XIII(1988)-XIV(1989)". En: The Yearbook on Commercial Arbitration, vol. XIV, 1989, pp. 534-555; y, Leonard V. Quigley, "Accession by the United States to the United Nations Convention on the Recognition and Enforcement of Foreign Arbitral Awards". En: The Yale Law Journal, vol, 70, nro. 7, 196t, pp. 1059-1060.

6. La lista actualizada de paises miembros puede ubicarse en: Comisión de las Naciones Unidas para el Derecho Mercantil Internacional (CNUMDI), Situación de las Convenciones y Leyes Modelo, Www.uncitral.org/spanish/status-s.htm.

7. Frente a una cada vez mayor masificación de causas en el Poder Judicial, el arbitraje ofrece a las partes una via mucho más expeditiva, menos formalista y que se adecua con mayor facilidad a las necesidades y expectativas de las partes.

Además, el arbitraje permite que actúen como árbitros expertos en la materia en discusión, a diferencia del proceso judicial en el que al juez muchas veces se le obliga a ser un "todista". De esta maneta, el arbitraje ofrece la excelente oportunidad de que la controversia sea resuelta por una o más personas especializadas en la materia en conflicto. Esta posibilidad que brinda el arbitraje de poder escoger a las personas que van a resolver un conflicto lo hace en especial atractivo.

A estas ventajas, cabe añadir la privaciđad y un ambiente de menor confrontación que el que aguarda a las partes en un proceso judicial.

Por último, el laudo arbitral es tan efectivo y ejecutable como una sentencia judicial.

Sobre el particular, leer a: Fernando Cantuarias Salaverry, "Arbitraje Comercial y de las Inversiones", ob. cit., pp. 3-12; Leonard L. Riskin \& James E. Westbrook, "Dispute Resolution and Lawyers", West Publishing, Minnesota, 1987, pp. 3 y ss.; Gilberto Peña Castrillón y Néstor Martinez Neira, "Pacto Arbitral y Arbitramento en Conciencia", Themis, Bogotá, 1986, pp. 6 y ss.; Nils Mangard, "El Arbitraje y el Sistema Judicial". En: Estudios sobre Arbitraje Comercial Internacional, Centro de Estudios Comerciales (CECO), Madrid, 1983, pp. 95 y ss.; Hans Smit, "The Future of International Commercial Arbitration: A single Transnational Institution? En: Columbia Journal of Transnational Low, vol. 25, nro. 1, 1986, pp. 15 y ss;; y, Warren E. Burger, "Isn't There a Better Way?". En: ABA Law Journal, American Bar Association, vol. 68, 1982, pp. 277 y ss. 
a) Las partes de una transacción comercial internacional suelen valorar la neutralidad, que difícilmente se obtiene teniendo que dirimir las controversias ante el Poder Judicial de una de las partes, ya que probablemente el proceso judicial se desarrollará en otro idioma, bajo reglas de procedimiento poco familiares, se requerirá el asesoramiento de abogados locales y, además, siempre existirá el riesgo de que los jueces discriminen en favor de su nacional. 8

En cambio, el arbitraje sí ofrece una instancia neutral para la solución de los conflictos que se generan de la contratación comercial internacional. ${ }^{9}$
b) Aun cuando se pacte la sumisión de una controversia ante un determinado Poder Judicial, siempre existe el riesgo que

8. Roque J. Caivano, "El Arbitraje y los Procesos de Integración (Su futuro en el Metcosur)". En: Revista Jurisprudencia Argentina, t. 1996-II, Buenos Aires, 1996, p. 791. "Si en cuestiones exclusivamente domésticas el arbitraje se ha revelado como una de las fórmulas más satisfactorias, sus ventajas se potencian cuando el conflicto involucra a partes radicadas en diferentes latitudes. La disyuntiva de acudir a una u otra jurisdicción judicial -reciprocamente teñidas de sospecha y parcialidad por la otra parte- es sólo subsanable mediante el recurso a un arbittraje neutral y especializado, en el que las partes pueden acordar no sólo la elección de la persona más idónea para el caso, sino también el lugar donde habrá de realizarse, el idioma, el procedimiento que los árbitros deberán seguir y aún el derecho de fondo aplicable". Gary B. Bom, "Forum selection versus Arbitration". En: International Commercial Litigation, vol. 39,1999, p. 30. "[N]ational courts inevitably apply local procedural rules to international disputes, which may be ill-suited for parties from different legal traditions and regions of the globe". Juan A. Cremades Sanz-Pastor, "La neuttalidad en el Arbitraje: La lengua, el lugar y la ley aplicable al Arbitraje". En: Estudios sobre Arbitraje Comercial Internacional, Centro de Estudios Comerciales (CECO), 2da Ed., 1983, p. 46, "en la mayor generalidad de los casos, las partes... van a un arbitraje comercial internacional... para obtener la neutralidad.

Neutralidad, no en el sentido de que el juzgador sea imparcial: esta imparcialidad también la encuentran ante la jurisdicción nacional. Neutralidad, en el sentido de igualdad de las partes ante el juzgador.

Efectivamente, una parte está siempre favorecida ante su juez nacional, por muy imparcial que sea éste. En efecto, para ganar un procedimiento se requiere demostrar que se tiene razón, y en esta demostración, la comunicación juega un papel importante.

Una parte puede comunicar mucho mejor ante un juez nacional que ante un juez extranjero: existen una serie de razonamientos, una identidad de puntos de vista, una similitud de raciocinio juridico que hacen que una parte sintonice mucho más fácilmente con su juez nacional que con un juez extranjero".

9. William W. Park, "Finality and Faimess in Tax Arbitration". En: Journal of International Arbitration, vol. 11, nro. 2, 1994, p. 20. "[T] he principal raison d'etre of international commercial arbitration has long been the enhancement of political and procedural neutrality... When a company in Boston concludes a joint venture with an Algerian State agency, neither party wants to end up litigating in the other side's bome court". 
otras jurisdicciones no reconozcan ese acuerdo y pretendan que sean ellas las llamadas a solucionar el conflicto. ${ }^{10}$

En otras palabras, existe el riesgo permanente de múltiples litigios judiciales, con los costos e incertidumbre que ello implica. ${ }^{11}$

Pero, además, puede suceder, como explica Caivano ${ }^{12}$, que "aun cuando [las partes] pudieran ponerse de acuerdo... y convenir someter sus asuntos a una determinada jurisdicción estatal... Ip]odría darse el supuesto de que la legislación del Estado elegido rechace el caso por considerarlo ajeno a su jurisdicción". 13

A todo esto hay que agregar, que hasta la fecha no existe una solución aceptable en el ámbito del Derecho Internacional, que reduzca la incertidumbre generada de la posibilidad de que los poderes judiciales se nieguen a respetar el pacto de sumisión acordado por las partes. ${ }^{14}$

10. Garrigues \& Andersen, Abogados y Asesores Tributarios, "El Arbitraje como fórmula alternativa de solución de conflictos. ¿Cuándo y cómo acudir al Arbitraje?" En: Boletin de Información Procesal y de Arbitraje, Madrid, 1999, p. 5. "[C]uando a la relación comercial se le añade un componente internacional, ya sea por la presencia de un socio extranjero o de negocios de 'export-import' de bienes y servicios, los escenarios de un posible litigio se multiplican en proporción directa al número de jurisdicciones estatales involucradas en la relación contrachual".

11. Roque J. Caivano, "Arbitraje: Su eficacia como sistema alternativo de resolución de conflictos", AdHoc, Buenos Aires, 1993, p. 86. "Podria darse el supuesto de que... las normas del pais de quien ha sometido sus asuntos a la jurisdicción de otro no permitan esta prórroga de jurisdicción y la reclamen para si".

12. Ibídem, p. 86 .

13. Peter D. Ehrenhaft, "Effective International Commercial Arbitration". En: Law and Policy in International Business, vol. 9, nro. 4, 1977, p. 1192. "Even if the contract contains both choice of law and choice of forum clauses, the judicial road to resolution of disputes remains full of obstacles. The selected forum may decline jurisdiction over the dispute if adjudication at that location would cause undue hardship to one of the parties, or if there is an insufficient relationship between the forum nation's law and the transaction out of which the dispute arose. Even if the selected court were willing to decide the dispute, that nation's law on conflict of laws might direct the parties to another body of law and forum altogether. Also, where the fonm selected by or for the parties is foreign to one or both, the procedures and substantive law may be unfamiliar".

[4. Gary B. Born, "International Arbitration and Forum Selection Agreements: Planning, Drafting and Enforcing", Kluwer Law International, The Hague, 1999, pp. 89-90. "Many developed countries, including the United States, are not party to any international treaty or other agreement relating to the enforcement of forum selection clauses. There are some bilateral or regional treaties governing international fonm agreements, such as the Brussels and Lugano Conventions, but most international forum selection clauses are governed in most national courts by domestic law". 


\title{
Estos y muchos otros problemas ${ }^{15}$ se reducen dramática- mente cuando se pacta la sumisión de las controversias a arbitra- je, ${ }^{16}$ ya que la mayoría de las veces es posible aplicar la Convención de Nueva York de 1958.17
}

\author{
c) Por último, conviene recordar que todavía no existe a \\ nivel mundial tratado alguno que permita la ejecución rápida, \\ segura y poco costosa de las sentencias judiciales. Ante esta \\ situación, muchas veces habrá que estar a lo que determine la \\ legislación de cada uno de los Estados en los que se pretenda eje- \\ cutar un fallo judicial, con los riesgos que ello implica. ${ }^{18}$
}

15. Como es el caso de la "jurisơicción exorbitante" o "brazo largo de la jurisdicción", mediante la cual algunos Estados amplían de manera intolerable la jurisdicción de sus cortes nacionales para conocer determinadas controversias. Sobre este tema, leer a: Joseph Halpem, "Exhorbitant Jurisdiction and the Brussels Convention: Toward a Theory of Restraint". En: The Yale Journal of World Public Order, vol. 9, nro. 1, 1982, pp. 369 y ss.; Beverly M. Carl, "La competencia en el Derecho Internacional Privado de los Estados Unidos". En: Derecho, nro. 40, Facultad de Derecho, Pontificia Universidad Católica del Perú, Lima, 1986, pp. 75 y ss.; y, Alan C. Swan \& John F. Murphy, "Cases and Materials on the Regulation of Internationat Business and Economic Relations", Matthew Bender, 1991, p. 866.

16. Gary B. Born, "Internationat Arbitration and Fonum Selection Agreements: Planning, Drafting and Enforcing", ob. cit., p. 13. "Similarly, "public policy' or 'mandatory law' limitations are usually less significant in arbitral that in judicial proceedings. For these reasons, it is often easier to obtain effective enforcement of an international arbitration agreement than of a forum selection clause".

17. Articulo II(3) de la Convención de Nueva York: "El Tribunal de uno de los Estados Contratantes al que se someta un litigio respecto del cual las partes hayan concluido un acuerdo en el sentido del presente articulo, remitirá a las partes al arbitraje, a instancia de una de ellas, a menos que compruebe que dicho acuerdo es nulo, ineficaz o inaplicable". Sobre este particular, leer a: Albert Jan van den Berg, "The New York Convention 1958: Consolidated Commentary, Cases reported in Volumes XIII(1988)-XIV(1989). En: Yearbook Commercial Arbitration, vol. XIV, 1989, pp. 557-565; Tom Carbonneau, "Cases and Materials on Commercial Arbitration", vol. 1, Juris Publishing, New York, 1997, p. 423; Gary B. Born, "International Commercial Arbitration in the United States", Kliwer Law and Taxation Publishers, Deventon \& Boston, 1994, pp. 285-359; Alejandro M. Garo, "Enforcement of Arbitration Agreements and Jurisdiction of Arbitral Tribunals in Latin America". En: Journat of International Arbitration, vol 1, nro. 4, 1989, p. 319; y, Albert Jan van den Berg, "Nondomestic Arbitral Awards under the 1958 New York Convention". En: Arbitration International, vol. 2, nro. 3, 1986, pp. 51-52.

18. Gary B. Born, "International Arbitration and Forum Selection Agreements: Planning, Drafting and Enforcing", ob. cit., pp. 106-107. "[A] substantial number of countries will generally not enforce foreign court judgments. Many states apply rules which deny recognition to any foreign judgment absent a treaty relationship with the rendering state (or 'state of origin') providing for mutual recognition and enforcement of judgments... Other countries simply refuse to recognize any foreign judgments, or admit foreign judgments solely as evidence in support of a party's substantive claims, which must be relitigated.

Even in countries where it is theoretically possible, the recognition and enforcement of foreign judyments is infrequently sought and even more rarely obtained. This is particularly true with respect to judgments against local nationals-against whom enforcement is typically most important. The enfor- 
En cambio, en el ámbito del arbitraje existe como hemos mencionado la Convención de Nueva York de $1958,{ }^{19}$ la cual permite el reconocimiento y la ejecución de laudos arbitrales en prácticamente todo el mundo. ${ }^{20}$

En consecuencia, la manera de asegurar que las controversias generadas del comercio internacional no sufran conflictos de competencia, terminen en manos de las Cortes de brazo largo o tengan finalmente problemas de ejecución de las sentencias, ${ }^{21}$ es pactando la sumisión de los futuros o actuales conflictos al arbitraje.

Por tanto, si en Latinoamérica realmente queremos fomentar el desarrollo masivo del comercio entre nuestras fronteras, necesariamente tenemos que garantizar que los comerciantes y empresarios puedan someter sus conflictos al arbitraje en cualquiera de nuestros países. ¿Es eso actualmente posible?

cement of foreign judgments can be subject to procedural delays and other shortcomings, especially in states where courts lack experience with such efforts. The absence of treaty commitments means that there are few external checks on parochial obstacles to enforcement against local nationals". Sobte el particular, leer a: José Daniel Amado, "Recognition and Enforcement of Foreign Judgments in Latin American Countries: An overview and update". En: Virginia Journal of International Law, vol. 31, nro. 1, 1990.

19. El Artículo 1(1) de este Tratado, dispone lo siguiente: "La presente Convención se aplicatá al reconocimiento y la ejecución de las sentencias arbitrales dictadas en el territorio de un Estado distinto de aquél en que se pide el reconocimiento y ejecución de dichas sentencias, y que tengan su origen en diferencias entre personas naturales o juridicas".

20. William Laurence Craig "Uses and Abuses of Appeal from Awards". En: Arbitration International, vol. 4, nro. 3, 1988, p. 174. "International Arbitration has become the ordinary way of resolving international commercial disputes. One of the reasons for this success has been the relative ease with which awards rendered in a foreign jurisdiction can be enforced at the debtor's domicile or in any jurisdiction where the debtor has assets. That ease has been due, in large part, to the New York Convention of 1958".

21. Pierre-Yves Gunter, "Enforcing Arbitral Awards, Injunctions and Orders". En: The Arbitration and Dispute Resolution Law Joumal, Part 4, 1999, pp. 265-266. "According to the leading commentator of the New York Convention, Professor Albert Jan van den Berg, the enforcement of an arbitral award has been refused in less than 5 per cent of the cases". 


\section{El Arbitraje en américa Latina}

Tradicionalmente Latinoamérica ha sido considerada como un subcontinente hostil al arbitraje. ${ }^{22}$

Esta afirmación resulta fácil de demostrar, simplemente apelando a las pobrísimas legislaciones arbitrales que nos han regido hasta hace muy pocos años ${ }^{23}$ y a la casi nula aceptación de tratados arbitrales universales, como son la Convención de Nueva York y la Convención sobre Arreglo de Diferencias Relativas a Inversiones entre Estados y Nacionales de otros Estados (CIADI). ${ }^{24}$

Sin embargo, a partir de la década pasada, las cosas han comenzado a cambiar para bien. 25

22. Ana I. Piaggi, "Evoluciones recientes del arbitraje comercial internacional en Latinoamérica". En: Revista de Derecho del Mercosur, nfo. 6, 2000, p. 148. "Nucstros paises son conocidos como un área tradicionalmente dificil para el arbitraje, incluso hostil, y somos probablemente la región del mundo que más lentamente accptó esta técnica como método de resolución de disputas". Sobre cl particular, leer a: Frank E. Nattier, "International Commercial Arbitration in Latin America: Enforcement of Arbitral Agreements and Awards". En: Texas International Law Journal, vol. 21, 1986, pp. 399 y ss.

23. Alejandro M. Garro, "El Arbitraje en la Ley Modelo propuesta por la Comisión de las Naciones Unidas para el Derecho Mercantil Internacional y en la Nucva Legislación Española de Arbitraje Privado: Un Modelo para la Reforma del Arbitraje Comercial en América Central". En: Arbitraje Comercial y Laboral en América Central, Alcjandro M. Garro (Ed.), Transnational Juris Publications, Nueva York, 1990; Alejandro M. Garro, "The UNCITRAL Model Law and the 1988 Spanish Arbitration Act: Models for reform in Central America". En: The American Review of International Arbitration, vol. 1, nro. 2, 1990; Alejandro M. Garro, "Enforcement of Arbitration Agreements and Jurisdiction of Arbitral Tribunals in Latin America", ob. cit.; y, Horacio Grigucra Naón, "Arbitration in Latin America: Overcoming traditional hostility". En: Arbitration International, vol. 5, nro. 2, 1989.

24. Recordemos la posición que asumieron los países latinoamericanos en 1965, en contra de la suscripción de la Convención del CLADI, cuyo pronunciamiento conjunto es conocido como el "No de Tokio". Sobre el particular, leer a: Michacl M. Moore, "International Arbitration between Statcs and Foreign Investors -The World Bank Convention". En: Stanford Law Review, vol. 18, nro. 6, 1966, p. 1376; Paul C. Szasz, "The Investment Disputes Convention and Latin America". En: Virginia Journal of International Law, vol. 11, nro. 2, 1971, pp. 256-265; y, Alden F. Abbott, "Latin Amcrican and International Arbitration Conventions: The Quandary of Non-Ratification". En: Harvard International Law Journal, vol. 17, 1976.

25. Fernando Mantilla Serrano, Major Trends in Intemational Commercial Arbitration in Latín America". En: Joumal of International Arbitration, vol. 17, nro. 1, 2000, p. 139. "Latin America can no longer be said to suffer from hostility towards intenational arbitration". Nigel Blackaby y Sylvia Noury, "International Arbitration in Latin America". En: LatinLawyer Review -Arbitration, 2007, p. 1. "Latin America has definitively shed its previously isolationist policies in the field of international dispute resolution". 
En efecto, a la fecha Antigua y Barbuda, Argentina, Bahamas, Barbados, Bolivia, Brasil, Colombia, Costa Rica, Cuba, Chile, Dominica, Ecuador, El Salvador, Guatemala, Haití, Honduras, Jamaica, México, Nicaragua, Panamá, Paraguay, el Perú, República Dominicana, San Vicente y las Granadinas, Trinidad y Tobago, Uruguay y Venezuela, son parte de la Convención de Nueva York. ${ }^{26}$

También la Convención sobre Arreglo de Diferencias Relativas a Inversiones entre Estados y Nacionales de otros Estados (CIADI), que regula el acceso al arbitraje para la solución de conflictos entre inversionistas y Estados receptores de inversión, cuenta hoy entre sus miembros con un número importante de Estados latinoamericanos. ${ }^{27}$

26. Por su parte, Argentina, Bolivia, Brasil, Chile, Colombia, Costa Rica, Eccuador, El Salvador, Guatemala, Honduras, México, Nicaragua, Panamá, Paraguay, el Perú, Unuguay y Venezuela han ratificado el Convenio Interameticano sobre Arbitraje Comercial Internacional (más conocido como la Convención đe Panamá de 1975). Este, sin embargo, es un Tratado de menor importancia y jerarquia frente al Convenio de Nueva York. Sobre este particular, leer a: Fernando Cantuarias Salaveny, "Arbitraje Comercial y de las Inversiones", ob. cit., pp. 424-432; Albert Jan Van Den Berg, "The New York Convention 1958 and Panama Convention 1975: Redundancy or Compatibility?" En: Arbitration International, vol. 5, nro. 3, 1989; Robert B. von Mehren, "The Enforcement of Arbitral Awards under Conventions and United States Law". En: The Yale Journal of World Public Order, vol. 9, nro. 1, 1982, p. 346; Gary B. Born, "International Arbitration and Fortm Selection Agreements: Planning, Drafting and Enforcing", ob. cit., p. 99; y, Horacio Griguera Naón, "Países de América Latina como Sede de Arbitrajes Comerciales Internacionales". En: Boletin de la Corte Internacional de Arbitraje de la CCI-El Arbitraje Cometcial Internacional, Suplemento Especial, 1995, p. 49.

27. A octubre de 2008, 142 Estados forman parte del Tratado, entre los que figuran un gran número de Estados latinoamericanos: Argentina (1994), Bahamas (1995), Barbados (1983), Colombia (1997), Costa Rica (1993), Chile (1991), Ecuador (1986), El Salvador (1984), Granada (1991), Guatemala (2003), Guyana (1969), Honduras (1989), Jamaica (1966), Nicaragua (1995), Panamá (1996), Paraguay (1983), el Perú (1993), San Vicente y las Granadinas (2003), Trinidad y Tobago (1967), Unuguay (2000) y Venezuela (1995). Ibrahim F.I. Shibata \& Antonio R. Parra, "The Experience of the International Centre for Settlement of Investment Disputes". En: ICSID Review -Foreign Investment Low Journal, vol. 14, nro. 2, 1999, p. 316. "Particularly impressive has been the increase in the number of ICSID members in Latin America, from two at the beginning of the period to thirteen today". Fernando Mantilla Serrano, "Major Trends in International Commercial Arbitration in Latin America", ob. cit., p. 139. "Concerning political risks and protection of foreign investment, the ratification of the 1965 World Bank Convention on the Settlement of Investment Disputes has contributed to reassure potential investors in the region". Sin embargo, Bolivia ha sido el primer Estado que ha denunciado este Tratado (mayo de 2007). Sobre este particular leer a: Julien Fouret, "Denunciation of the Washington Convention and Non-Contractual Investment Arbitration: 'Manufacturing Consent" to ICSID Arbitration?". En: Joumal of International Arbitration, Vol. 25, No. 1, 2008, pp. 71-87; Jorge Luis Inchauste y Ratniro Guevara, "Bolivia's Withdrawal from ICSID". En: Global Asbitration Review -The Arbitration Review of the Americas 2008, www,globalarbitrationreview.com/handbooks/4/sections/8/chapters/53/bolivia; y Christian Leathley, "Morales about to take on the Constitutional Tribunal?" En: Global Arbitration Review, Vol. 2, No. 3, 2007, pp. 13-16. 


\section{Además, como identifican Craig, Park \& Paulsson, ${ }^{28}$ cada vez son más las partes que provienen de Latinoamérica que intervienen en arbitrajes ante la Cámara de Comercio Internacional (CCI), principal centro de arbitraje comercial del mundo.}

Por último, si bien es cierto que al momento en que se escriben estas líneas, las legislaciones arbitrales de Argentina, ${ }^{29}$

28. W. Laurence Craig, Willian W. Park \& Jan Paulsson, "International Chamber of Commence Arbitration", 3era. Ed., Oceana Publications/ICC Publishing, 2000, p. 5. "Latin American countries seem to be overcoming much of their traditional resistance to international arbitration. Parties from Latin America, who constituted only $3.8 \%$ of the ICC users in 1987, represented $9.8 \%$ in 1999 . Latin America is now the fourth most frequently represented region in ICC arbitration after Westem Europe, North America and the Far East. This included twelve countries with more than five nationals represented in ICC abitral proceedings".

Los autores en la Tabla No. 5 (pp. 732-733) de su colosal obra identifican que de 1989 a 1999, 241 empresas o personas latinoamericanas participaron como demandantes y 306 como demandados en arbitrajes ante la CCI, destacando por su número partes de México, Panamá, Argentina, Brasil y Venezuela, en ese orden.

Paul E. Mason y Mauricio Gomm-Santos, "New Keys to Arbitration in Latin-America". En: Joumal of International Arbitration, Vol, 25, No. 1, 2008, pp. 46-47. "Latin America bas seen phenomenal growth in institutional arbitration over the last decade. Cases from Latin America in ICSID, the ICDR/AAA, ICC, WIPO and LClA have been growing at rising rates".

29. Respecto a la legislación arbitral argentina, Horacio A. Griguera Naón, "Recent Trends Regarding Commercial Arbitration in Latin America", ob. cit., pp. 100-101, la critica abiertamente, al afirmat que "legal reform has not reached out yet to all Latin American national jurisdictions. Argentina is an example in spite of several past or presently ongoing attempts to introduce or pass legislation to overcome the present unsatisfactory situation". En el año 2001, el Ministerio de Justicia presentó al Congreso Federal un proyecto de Ley Federal de Arbitraje desarrollado a partir de la Ley Modelo de UNCITRAL. Sin embargo, hasta ef momento en que se escriben estas lineas, el proyecto sigue encarpetado en el Congreso. Para una revisión de la actual legislación arbitral argentina, como del proyecto de Ley Federal de Aibitraje, recomendamos leer a: Alessandro Spinillo y Emilio Vogelius, "Argentina". En: International Arbitration in Latin America, Nigel Blackaby, David Lindsey y Alessandro Spinillo (editores), KLuwer Law International, La Haya, 2002, pp. 17-59; María Beatriz Burghetto, "Current Status of Arbitration Legislation in Argentina". En: Journal of International Arbitration, vol. 21, nro. 6, 2004, pp. 479-498; y Femando Aguilar, "¿Para qué sirve una ley nacional de arbitraje?".

En: La Ley, www.laleyonline.com.at/app/laley/documentBody?num2re=20\&collection. La Ley Federal de Arbitraje se ubica en: www.sice.oas.org/DISPUTE/COMARB/argentina/libro6.asp. Por su parte, el proyecto de Ley Federal de Arbittaje se ubica en: www.servilex.com.pe/arbitraje/argentina/ley_pdf.pdf.

Las cortes argentinas han emitido en los últimos años una serie de fallos (Cartellone, Yacyretá, National Grid, entre otros) que preocupan significativamente por su poca (acaso nula) actitud en pro del arbitraje. Sobre este particular, leer a: Pablo F. Richards y Guido Barbarosch, "Argentina". En Global Arbitration Review -The Arbitration Review of The Americas 2008, www.globalarbitrationreview.com/handbooks/4/sections/8/chapters/48/argentina; y Paul E. Mason y Mauricio GommSantos, "New Keys to Artitration in Latin-America" ob. cit., pp. 33-34.

322 Revista ECUATORIANA De ARbitraJE 
República Dominicana, ${ }^{30}$ Trinidad y Tobago ${ }^{31}$ y Uruguay, ${ }^{32}$ entre otras, ${ }^{33}$ mantienen disposiciones arbitrales anticuadas y poco amigables para la práctica del arbitraje, donde más se aprecia el cambio en pro del arbitraje es en el número importante de recientes legislaciones arbitrales dictadas en más de una docena de países en poco más de una década. A saber: Bermuda (1993), Bolivia (1997), Brasil (1996), Colombia (1998), Costa Rica (1997), Chile (2004), Ecuador (1997), El Salvador (2002), Guatemala (1995), Haití (2006), Honduras (2000), México (1993), Nicaragua (2005), Panamá (1999), Paraguay (2000), el Perú (1996/2008) y Venezuela (1998). ${ }^{34}$

30. Las disposiciones arbitrales de República Dominicana contenidas en el Código de Procedimientos Civiles (Libro III), modificado por la Ley 845 de 15 de julio de 1978, se ubican en: www.servilex.com.pe/arbitraje/repdominicana/legrd.html. Sobre este particular, leer a: Rafael E. Cáceres R., "Experiencia dominicana en arbitraje". En: Revista Jberoamericana de Arbitraje, www.servilex.com.pe/arbitraje/repdominicana/artrcrd.html.

31. En lo que se refiere a la legislación atbitral de Trinidad y Tobago (1939), leer. "ALCA. Grupo de Negociación sobre Solución de Controversias. Cuestionario", www.ftaa.alca.org/busfac/comarb/trinidad/questris.asp, pp. 1-5.

32. Las disposiciones sobre arbitraje contenidas en la Ley 15.982, Código General del Proceso (Título VII) del Uruguay, se ubican en: www.sice.oas.org/DISPUTE/COMARB/Unguay/LI5982s.asp. El 25 de mayo de 2004, el Ejecutivo uruguayo remitió al Congreso un proyecto de Ley sobre Arbitraje Comercial Internacional. Sin embargo, hasta el momento en que se culminó este trabajo no habia sido promulgado. Sobre este particular, leer a: Sandra González, "Unuguay". En: Global Arbitration Review -The Arbitration Review of The Americas 2008, www.globalarbitrationreview,com/handbooks/4/sections/8/chapters/57/uruguay.

33. El Centro de Estudios de Justicia de las Américas (www.cejamericas.org/reporte/muestra pais.php) y el SICE (www.sice.oas.org/DISPUTE/COMARB/canale.asp) no identifican la existencia de legislaciones arbitrales en Antigua y Barbuda, Barbados, Belice, Dominica, Granada, Guyana, Jamaica, Santa Lucía, San Vicente y las Granadinas, St. Kitts and Nevis y Surinam. Sin embargo, la Ley de Asbitraje de Guyana (de 1916, reformada en 1929 y 1953) y la Ley de Artitraje de Belice (de 1926, reformada en 1932) se ubican en: www.gina.gov.gy/gina-pub/Laws/cap703.pdf y www.belizelaw.org/lawadmin/pdf\%20files/cap 125.pdf, respectivamente.

34. Horacio A. Griguera Naón, "Recent Trends Regarding Commercial Arbitration in Latin America", documento presentado en el First Annual Miami International Arbitration Conference, ICDR International Centre for Dispute Resolution y Stell Hector Davis International, Miami, 2003, pp. 95 96. "There is no doubt -it has by now become almost truism- that Latin America has become a more fertile ground than before for the development of commercial arbitration [...] one of the dominant characteristics of the evolution of commercial arbitration in Latin America during the last ten years has been the proliferation of new legislation regarding commercial arbitration in this part of the world". Sobre el particular, leer a: Thomas E. Carbonneau, "The Ballad of Transborder Arbitration", ob. cit., pp. 783-785. 


\section{II. ¿Existe un Marco legal Adecuado para la Práctica del Arbitraje Comercial Internacional en la Región?}

Creemos que no, como trataremos de demostrar seguidamente, agrupando para el efecto a las leyes arbitrales en cuatro categorías.

\section{II.1 Legislaciones que no distinguen entre arbitrajes nacionales e internacionales y que, además, sus disposiciones son tan localistas y alejadas de los estándares internacionales, que resultan muy poco amigables para la práctica del arbitraje comercial internacional dentro de sus fronteras 35}

En este grupo ubicamos a las legislaciones arbitrales de Brasil, Costa Rica y Venezuela.

El caso de Brasil es digno de destacar, ya que si bien cuenta con una reciente legislación arbitral -Ley de Arbitraje 9307- no distingue entre arbitraje nacional y arbitraje internacional ${ }^{36} \mathrm{y}$, además, aún mantiene disposiciones poco modernas. ${ }^{37}$

Esta ley tiene serios errores, como el de mantener, por lo menos en parte, la clásica distinción entre cláusula compromisoria y compromiso arbitral. ${ }^{38}$ Además, como explica Bosco, ${ }^{39}$ exis-

35. Nigel Blackaby, David M. Lindsey y Alessandro Spinillo, "Overview of Regional Development", ob. cit., p. 7. "Unfortunately, several countries did not opt for the Model Law but insisted on developing their own projects. They took certain elements of the Model Law but introduced aspects of their own historic arbitral procedures which they considered appropriate or which raised issues of public policy, without examining the effect of these elements on foreign parties. The countries which fall within tis category include Brazil (Law of 1996), Colombia (Law of 1996) and Costa Rica (Law of 1997)".

36. En nuestra opinion, este solo hecho convierte al Brasil en un foro arbitral internacional muy poco atractivo.

37. La Ley de Arbitraje se ubica en: www.servilex.com.pe/arbitraje/brasil/leyarbbra.html. Sobre el tema, leer a: Carlos Nehring Netto, "The New Brazilian Arbitration Law". En: The ICC International Court of Arbitration Bulletin -International Commercial Arbitration in Latin America, Special Supplement, 1997, pp. 11-14.

38. Horacio A. Griguera Naón, "Recent Trends Regarding Commercial Arbitration in Latin America", ob. cit., p. 101. "[T]he new Brazilian Act maintains the "compromiso' (apparently... on the basis of an interpretation of Article 5 of this statute a 'compromiso' is not required when the partics have agreed on institutional arbitration or on the application of an ad-hoc arbitration rules excluding the "compromiso')". Sobre el tema, leer a: Carlos Nehring Netto, "The New Brazilian Arbitration Law". En; The 
te falta de claridad acerca de la aplicación del principio de la competencia de los árbitros para definir su propia competencia y no existe norma alguna que regule la forma de determinar la ley aplicable al fondo de la controversia.

Se trata pues de una ley de arbitraje poco moderna y alejada significativamente de los estándares internacionales, por lo que resulta muy poco amigable para la práctica del arbitraje internacional. Es más, nos atrevemos a afirmar que inclusive limita gravemente el desarrollo del arbitraje doméstico. ${ }^{40}$

El caso de Costa Rica es similar al de Brasil, ya que se trata de una ley de arbitraje absolutamente localista y con ninguna vocación para atraer conflictos internacionales a sus fronteras.

En efecto, Costa Rica aprobó su Ley sobre Resolución Alterna de Conflictos y Promoción de la Paz Social 7727 en 1997. La norma, si bien ha significado una importante mejora en las reglas de juego sobre arbitraje en comparación con el anterior marco legal existente en ese país, lo cierto es que no cuenta con reglas especiales en materia de arbitraje internacional, obligando por tanto a arbitrar bajo disposiciones domésticas muy poco amigables. ${ }^{41}$

Este también es el caso de Venezuela, país que, si bien cuenta con una reciente Ley de Arbitraje Comercial 26.43042, posee

ICC International Court of Arbitration Bulletin -International Commercial Arbitration in Latin America, Spectal Supplement, 1997, pp. 11-14.

39. Joao Bosco Lee, "Brazil". En: International Arbitration in Latin America, Nigel Blackaby, David Lindsey y Alessandro Spinillo (editores), Kluwer Law International, La Haya, 2002, pp. 61-88.

40. Las cortes del Brasil han emitido en los últimos años una serie de fallos (Copel, Compagás, CEEE, entre otros) que si bien han estado referidos a la intervención de entidades y empresas públicas en arbitrajes, no dejan de preocupar, debido a la facilidad con la que las cortes se entrometen durante el desarrollo de los procesos arbitrales. Sobre este particular, leer a: Paul E. Mason y Mauricio GonmSantos, "New Keys to Arbitration in Latin-America" ob. cit., pp. 33-36.

41. La ley se ubica en: www.servilex.com.pe/arbitraje/costa rica/7727cr.html.

42. Aqui hay que tener mucho cuidado. Esta ley solo se aplica respecto a cuestiones que el Código de Comercio de Venezuela define como "comerciales". Si una controversia no es considerada "comercial", uno podría acabar arbitrando en Venezuela al amparo de normas arbitrales obsoletas contenidas en el Código Procesal Civil de ese país. Bernardo Weíninger y David M. Lindsey, "Venezuela". En: International Arbitration in Latin America, Nigel Blackaby, David Linđsey y Alessandro Spinillo 
disposiciones que tampoco distinguen entre arbitraje nacional y arbitraje internacional, $43 \mathrm{y}$, además, existen muchos vacíos $\mathrm{y}$ lagunas que habilitan la intervención judicial durante el procedimiento arbitral. 44

Es más, como explican Anzola y Zumbiehl, ${ }^{45}$ recientes fallos del Tribunal Supremo de Justicia demuestran una actitud muy poco amigable hacia el arbitraje. Así, por ejemplo, se ha desconocido la competencia de los árbitros para pronunciarse acerca de su propia competencia.

Estamos pues ante la presencia de una legislación y de una conducta jurisprudencial que no tienen como vocación atraer conflictos internacionales. 46

(editores), Kluwer Law International, La Haya, 2002, p. 230. "The new law... applies only to arbitration of disputes arising from commercial activities (Article 1 of the Artitration Law). Article 2 of the Venezueian Commercial Code... provides a list of several activities that are considered commercial. In addition, Article 3 of the Commercial Code contains a presumption that activities performed by merchants are deemed commercial activities. Non-commercial arbitration matters continue to be subject to... Civil Procedure Code arbitration rules...". La Ley de Arbitraje Comercial se ubica en: www.servilex.com.pe/arbitraje/venezuela/leyarve.html.

43. La ley como indican Ninfa Urdaneta y John H. Rooney, Jr., "The Law and Practice of International Commercial Arbitration in Venezuela". En: World Arbitration \& Mediation Report, vol. 11, nro. 1, 2000 , p. 20, "does not distinguish between international and domestic arbitration", lo que, en nuestra opinión, este solo hecho la hace poco amigable para la práctica del arbitraje internacional.

44. Bernardo Weininger, "Validity of arbitral agreement and enforcement of arbitral awards in Venezuela", documento presentado en el First Annual Miami International Arbitration Conference, ICDR International Centre for Dispute Resolution y Stell Hector Davis International, Miami, 2003, p. 5. "The Arbitration Law contains several loopholes that may result in the intervention of the judjciary during the course of an arbitral procedure. Hence, this shall be carefully reviewed before including any contractual provision submitting to any agreement to arbitrate under the rules of the Atbitration Law".

45. J. Eloy Anzola y Frédéric Zumbiehl, "El Tribunal Supremo de Venezuela riñe con el arbitraje", documento presentado en el Second Annual Conference "International Commercial Arbitration in Latin America: The ICC Perspective", International Chamber of Commerce, Miami, 2004, pp. 1-40.

46. Sobre este particular, leer a: Nigel Blackaby y Sylvia Noury, "International Arbitration in Latin America". En: Latin Lawyer Review, 2005, p. 2. 


\section{II.2 Legislaciones que si bien distinguen entre arbitrajes nacionales e internacionales, al aplicar al arbitraje internacional un número importante de disposiciones locales, terminan por convertir a esos foros en poco amigables para la práctica de arbitrajes comerciales internacionales}

Aquí el ejemplo más claro es Colombia, ${ }^{47}$ ya que aun cuando distingue entre arbitraje nacional y arbitraje internacional, 48 apenas cuenta con dos artículos aplicables a este último. ${ }^{49}$

Debido a la falta de reglas precisas para el desarrollo de arbitrajes internacionales, es de prever que serán de aplicación las normas sobre arbitraje nacional o doméstico, las cuales son sumamente localistas y se apartan significativamente de los estándares internacionales. 50

47. Colombia cuenta con el Decreto 2279 (1989), que ha sido modificado por la Ley 23 (1991), el Decreto 2651 (1991), Ja Ley 315 (1996) y la Ley 446 (1998). Todas estas disposiciones han sido consolidadas en el Estatuto de los Mecanismos Alternativos de Solución de Conflictos, Decreto 1818 (1998). El Decreto 1818, se ubica en: www.sice.oas.org/DISPUTE/COMARB/Colombia/D1818I.asp.

48. El attículo I de la Ley 315 (numeral 196 del Decreto 1818) dispone: "Setá internacional el arbitraje cuando las partes asi lo hubieren pactado, siempre que además se cumpla con cualquiera de los siguientes eventos:

1. Que las partes al momento de la celebración del pacto arbitral, tengan su domicilio en Estados diferentes.

2. Que el iugar de cumplimiento de aquella parte sustancial de las obligaciones directamente vinculadas con el objeto del litigio, se encuentre situada fuera del Estado en el cual las partes tienen su domicilio principal.

3. Cuando el tugar del arbitraje se encuentre fuera del Estado en que las partes tienen sus domicilios, siempre que se hubiere pactado tal eventualidad en el pacto arbitral.

4. Cuando la controversia sometida a decisión arbitral afecte directa e inequivocamente los intereses del comercio intemacional...".

49. El articulo 2 de la Ley 315 (numeral 197 del Decreto 1818), dispone: "El arbitraje internacional se regirá en todas sus partes de acuetdo con las normas de la presente ley, en particular por las disposiciones de los tratados, convenciones, protocolo y demás actos de derecho internacional suscritos y ratificados por Colombia, los cuales priman sobre las reglas que sobre el particular se establecen en el Código de Procedimiento Civil. En codo caso, las partes son libres de determinar la norma sustancial aplicable conforme a la cual los átbitros habrán de resolver el litigio. También podtán directamente o mediante referencia a un reglamento de arbitraje, determinar todo lo concerniente al proceditniento arbitral, incluyendo la convocatoria, la constitución, la tramitación, el idioma, la designación y nacionalidad de los árbitros, asi como la sede del tribunal, la cual podrá estat en Colombia o en un país extranjero". Sobre el particular, leer a: Marco Gerardo Monroy Cabra, "El atbitraje internacional en Colombia". En: Revista Iberoamericana de Arbitraje, www.servilex.com.pe/arbitraje/congresopanama/b-07.html; y Femando Mantilla-Serrano, "Colombian Arbitration Legislation". En: The ICC International Court of Arbitration Bulletin -International Commercial Arbitration in Latin America, Special Supplement, 1997, pp. 21-31.

50. Sobre este particular, leer a: Fernando Mantilla-Serrano, "Colombia". En: International Arbitration in Latin America, Nigel Blackaby, David Lindsey y Alessandro Spinillo (editores), KJuwer Law International, La Haya, 2002, pp. 11l-134. 
Por estas razones, nosotros consideramos que se trata de una plaza poco atractiva para la práctica del arbitraje internacional. 51

En la misma situación se encuentra Ecuador, ya que su Ley de Arbitraje y Mediación 145/97,52 si bien distingue entre arbitraje nacional y arbitraje internacional,,$^{53}$ no cuenta con regulación alguna respecto a este último..$^{54}$

En consecuencia, aquí también será previsible la aplicación de la normatividad doméstica, la cual es poco amigable para la práctica del arbitraje internacional. .55

En el mismo grupo ubicamos a las legislaciones arbitrales de Bolivia, ${ }^{56}$ El Salvador, ${ }^{57}$ Honduras, ${ }^{58}$ Panamá, ${ }^{59}$ Nicaragua $^{60}$ y

51. Al momento en que se escriben estas lineas, se viene discutiendo en el Congreso colombiano un proyecto de ley de arbitraje. Aun cuando el proyecto sigue de cerca la Ley Modelo de UNCITRAL, lamentablemente mantiene disposiciones poco amigables para la práctica del arbitraje internacional en ese pais.

52. La Ley de Arbitraje y Mediación se ubica en: www.servilex.com.pe/arbitraje/ecuador/leyarbitraje.html.

53. El artículo 41 dispone: "Sin perjuicio de lo dispuesto en los tratados internacionales un arbitraje podrá ser internacional cuando las partes así lo hubieren pactado, sienspre y cuando se cumplan cualquiera de los siguientes requisitos: a) Que las partes al momento de la celebración del convenio arbitral, tengan sus domicilios en estados diferentes; $o$, b) Cuando el lugar de cumplimiento de una parte sustancial de las obligaciones o el lugar en el cual el objeto del litigio tenga una relación más estrecha, está situado fuera del estado en que, por lo menos una de las partes, tiene su domicilio; $0, c$ ) Cuando el objeto del litigio se refiere a una operación de comercio intemacional".

54. El artículo 42 establece: "El arbitraje internacional quedará regulado por los tratados, convenciones, protocolos y demás actos de derecho internacional suscritos y ratificados por el Ecuador.

Toda persona natural 0 jurídica, pública o privada, sin restricción alguna es libre de estipular directamente o mediante referencia a un reglamento de arbitraje todo lo concemiente al procedimiento arbitral, incluyendo la constitución, la tramitación, el idioma, la legislación aplicable, la jurisdicción y la sede del tribunal, la cual podrá estar en el Ecuador o en país extranjero...".

55. Sobre el tema, leer a: Ramiro Salazar Cordero, "Ecuador". En: International Arbitration in Latin America, Nigel Blackaby, David Lindsey y Alessandro Spinillo (editores), Kluwer Law International, La Haya, 2002, pp. 135-154; Alfredo Larrea-Falcony, "Arbitration in Ecuador". En: The ICC International Court of Arbitration Bulletin -International Commercial Arbitration in Latin America, Special Stpplement, 1997, pp. 41-48; y Xavier Andrade Cadena, "Las ventajas del atbitraje internacional: una perspectiva ecuatoriana". En: Revista Iberoamericana de Arbitraje, www.servilex.com.pe/arbitraje/ colaboraciones/ventajas_internacional.html, pp. 5-14.

56. La Ley de Atbitraje y Conciliación 1770 cuenta con un título especial en materia de arbitraje intemacional. La ley considera internacional a un arbitraje desartollado en territorio boliviano, cuando: "Articulo 71.- I. A los efectos de la presente ley, un arbitraje será de carácter intemacional, en los casos siguientes:

1. Cuando al momento de celebrar el convenio arbitral, las partes tengan sus establecimientos en Estados diferentes. 
2. Cuando cl lugar de cumplimicnto de una parte sustancial de las obligaciones o el lugar con el cua] el objeto de la controversia tenga una relación mís estrecha se encuentre fuera del Estado en el que las partes tienen sus establecimientos.

3. Cuando las partes hubieren convenido expresamente que la materia arbitrable está relacionada con más de un Estado.

II. A los efectos de determinar el carácter intemacional de un arbitraje, cuando una de las partes tenga más de un establecimiento para el ejercioio de sus actividades principales, se considerará aquel que guarde relación con el convenio arbitral. Cuando una parte no tenga ningún establecimitento, se tomará en cuenta su residencia habitual". La ley se ubica en: www.servilex.com.pe/arbitraje/bolivia/leyarbo.html.

57. El artículo 3(h) de la Ley de Mediación, Conciliación y Arbitraje, Decreto 914-2002, dispone: "Arbitraje Internacional: El que se da en cualquiera de los siguientes casos:

1) Cuando las partes de un convenio arbitral tengan, al momento de celebración del mismo, sus domicilios en Estados diferentes.

2) Si uno de los lugares siguientes está situado fuera del estado en el que las partes tienen sus domicilios: a) El lugar del arbitraje, si este se ba determinado en el Convenio Arbitral, o con arreglo al mismo sea distinto.

b) El lugar del cumplimiento de una parte sustancial de las obligaciones de la relación jurídica o el lugar con el cual el objeto del lítigio tenga una relación más estrecha". La ley se ubica en: www.camarasal.com/leyespdf/LEY\%20DE $\% 20$ MEDIACION,\%20CONCILIACION\%20Y\%20AR BITRAJE.pdf,

58. El articulo 86 de ta Ley de Conciliación y Arbitraje, Decreto 161-2000, dispone: "El arbitraje es internacional en los siguientes casos:

1) Cuando las partes de un convenio arbitral tengan, al momento de celebración del mismo, sus domicilios en Estados diferentes.

2) Si uno de los lugares siguientes está situado fuera del estado en el que las partes tienen sus domicilios: a) El lugar del arbitraje, si este se ha determinado en el convenio arbitral o con arreglo al mismo sea distinto. b) El tugar del cumplimiento de una parte sustancial de las obligaciones de ta relación juridica o el lugar con el cual el objeto del litigio tenga una relación más estrecha". La ley se ubica en: www.biblio.unah.edu.hn/docum/arch/ley_de_conciliacion.pdf.

59. El artículo 5 de la Ley de Arbitraje y Mediación, Decreto Ley 5 de 1999, dispone: "El arbitraje comer" cial internacional es, de conformidad con el presente Decreto-Ley, cuando el objeto o negocio jurídico contenga elementos de extranjería o de conexión suficientemente significativos que lo caractericen como tal o bien que conforme a la regla de conflicto del foro lo califiquen como internacional. También se considerará que el arbitraje es comercial internacional al concurrir alguna de las circunstancias siguientes:

1. Si las partes en un convenio arbitral tienten, al momento de la celebración de ese convenio, sus establecimientos u oficinas en Estados diferentes.

2. Si el lugar de arbitraje que se ha determinado en el convenio arbitral o con arreglo a este, está situado fuera del pais en que las partes tienen sus establecimientos.

3. Si el lugar de cumplimiento de las obligaciones derivadas de la relación juridica que vincula a las partes, está situado fuera del país en que las partes tienen sus establecimientos.

4. Si el lugar con respecto al cual la controversia guarda una relación más estrecha, está situado fuera del pais en que las partes tienen sus establecimientos.

5. Si la materia objeto del arbitraje es de naluraleza civil o mercantil internacional y/o está relacionada con más de un Estado y/o consista en prestaciones de servicios, enajenación o disposición de bienes o transferencia de capitales que produzcan cfectos transfronterizos o extraterritoriales". La ley se ubica en: www.servilex.com.pe/arbitraje/panama/leyarpa.htmi.

60. El artículo 22 de la Ley de Mediación y Arbitraje, Ley 540 de 2005, dispone: "Un arbitraje será internacional cuando las partes en un acuerdo de arbitraje tienen, al momento de la celebración de ese acuerdo, sus respectivos domicilios en Estados diferentes.

También tendrá el carácter de arbitraje internacional cuando uno de los lugares enumerados a contimuación está situado fuera del Estado en que las partes tienen sus domicilios:

1. El lugar del arbitraje, si este se ha detenninado en el acuerdo de arbitraje o con arreglo al acuerdo de arbitraje.

2. El lugar del cumplimiento de una parte sustancial de las obligaciones de la relación comercial o el lugar con el cual el objeto de litigio tenga una relación más estrecha. [...]

También se reconocerá como arbitraje internacional cuando las partes han convenido expresamente en qque la cuestión objeto del acuerdo de arbitraje está relacionada con más de un Estado". La ley se ubica en: http://legislacion.asamblea.gob.ni/Normaweb.nsf(\$All)/D0489E6135592D16062570. 
Haitíb1, que tienen en común el mismo problema ya detectado, aunque sus disposiciones locales se apartan en menor grado de los estándares internacionales. Sin embargo, la sola existencia de estas normas locales hace poco atractiva la práctica del arbitraje internacional dentro de sus fronteras.

Este problema es reconocido, por ejemplo, por la doctrina panameña, la que identifica que aun cuando su ley de arbitraje ha recogido casi en su integridad la Ley Modelo de UNCITRAL, lo cierto es que se aplican diversas disposiciones locales que limitan significativamente la práctica del arbitraje internacional. 62

II.3 Legislaciones que aplican a todo arbitraje que se desarrolle dentro de sus fronteras los mismos estándares idóneos para la práctica del arbitraje internacional, al haber adoptado prácticamente en su integridad la Ley Modelo de UNCITRAL ${ }^{63}$

Nos referimos fundamentalmente a México ${ }^{64}$ y Perú $^{65}$ y, en menor medida y en ese orden, a Guatemala y Paraguay.

En efecto, México ha sido el primer Estado latinoamericano en modernizar su legislación sobre arbitraje, al haber realizado

61. Artículo 971 del Código de Procedimientos Civiles, según modificación de 2006: "Est international l'arbitrage qui met en cause des intérets du commerce international".

62. Sobre el tema, leer a: Gilberto Boutin, "La noción de arbitraje comercial internacional". En: Revista Iberoamericana de Arbitraje, www.servilex.com.pe/arbitraje/congresopanama/a-03.html; y Ulises Pitti G. "La regulación del arbitraje, la conciliación y la mediación en la legislación panameña". En: Revista lberoamericana de Arbitraje, www.servilex.com.pe/arbitraje/congresopanama/a-04.html.

63. Nigel Blackaby y Sylvia Noury, "Intemational Arbitration in Latin America", ob. cit, p. 2. "The arrival of the Model Law was well timed for Latin America: it offered legislators a means of implementing a new and modern legislation without the need to 'reinvent the wheel' and ensured that the lack of an arbitral tradition did not prejudice the quality of any new law. It was the best starting point for Latin America, which in general did not have such an arbitral traditjon".

64. Claus von Wobeser, "México". En: International Arbitration in Latin America, Nigel Blackaby, David Lindsey y Alessandro Spinillo (editores), Kluwer Law International, La Haya, 2002, p. 191. "Mexico's quick adoption of the Model Law and its clear policy stance in favour of arbitration has made it the jurisdiction of choice for hosting international arbitrations in Latin America".

65. Fernando Cantuarias Salaverry y Roque J. Caivano, "La Nueva Ley de Arbitraje Peruana: Un nuevo salto a la modernidad". En: Revista Peruana de Arbitraje, No. 7, Lima, 2008, pp. 43-84. 
modificaciones a las disposiciones arbitrales contenidas en el Código de Comercial Federal en el año de 1993,66 adoptando esencialmente la Ley Modelo de UNCITRAL. ${ }^{67}$

Sin embargo, cabe recordar que México es un Estado federal, ${ }^{68}$ por lo que esta moderna ley de arbitraje ${ }^{69}$ se aplicará exclusivamente cuando la materia controvertida sometida a arbitraje sea considerada comercial ${ }^{70}$ conforme a las disposiciones del Código de Comercio Federal. ${ }^{71}$

Caso contrario, uno correrá el riesgo de someter una controversia a arbitraje bajo regulaciones estatales muy poco amigables para la práctica del arbitraje internacional. ${ }^{72}$

66. Julio C. Trevifino, "International Commercial Arbitration in Mexico". En: The ICC International Court of Arbitration Bulletin -International Commercial Arbitration in Latin America, Special Supplement, 1997, p. 54. "The current legislation on commercial arbitration... was introduced by the Mexican Federal Congress as an amendment to the existing Federal Code of Commerce... as Title IV, Book V...".

67. Ibídem, p. 54. "The new law substantially incorporates the UNCITRAL Model Law of $1985 \ldots$.. plus several provisions taken from the UNCITRAL Arbitration Rules of 1976 regarding costs, and a few procedural nules".

68. Ibidem, p. 54. "Mexico is a Federal Republic composed of 31 states and a Federal District. Therefore, there is a dual system of federal and state laws and courts; and under the Federal Constitution, the Federal Congress has the exclusive jurisdiction to legislate on commercial matters, incliding commercial arbitration. Thus, the legislation on commercial arbitration in force, both domestic and international, is federal law and is applicable across the nation. Under certain circumstances, state procedural law may be applied to supplement the law on commercial arbitration". Claus von Wobeser, "México", ob. cit., p. 158. "Mexico's Constitution provides for a federal system of government which the Federal Congress has the authority to make laws in specific areas, such as commercial laws, including commercial arbitration. The state congresses may pass laws on the matters not reserved to the federal congress such as atbitration rules in civil matters".

69. El artículo 1416(III) del Código de Comercio Reformado de México (1993) contiene una definíción sobre arbitraje internacional, con el siguiente tenor: "Arbitraje internacional, aquel que:

a) Las partes al momento de la celebración del acuerdo de arbitraje, tengan sus establecimientos en países diferentes; 0

b) El lugar de arbitraje, determinado en el acuerdo de arbitraje o con arreglo al mismo, el lugar del cumplimiento de una parte sustancial de las obligaciones de la relación comercial o el lugar con el cual el objeto del litigio tenga una relación más estrecha, esté situado fuera del pais en el que las partes tienen su establecimiento". Sin embargo, las normas arbitrales se aplican por igual a todos los arbitrajes comerciales que se desarrollen en México, sean estos nacionales o internacionales.

70. El artículo 1415 del Código de Comercio Reformado de México ([993) dispone: "Las disposiciones del presente título se aplicarán al arbitraje comercial nacional, y al internacional cuando el lugar del arbitraje se encuentre en el territorio nacional...".

71. Francisco González de Cossío, "Prognosis de la salud arbirral mexicana", www.camex.com. mx/prognosis.pdf, realiza un análisis de la actitud de los tribunales judiciales mexicanos frente al arbitraje.

72. Sobre el tema, leer a: Claus von Wobeser, "México", ob. cit., pp. 155-191; y Julio C. Treviño, "International Commercial Arbitration in Mexico", ob. cit., pp. 54-60. La ley se ubica en: www.servilex.com.pe/arbitraje/legmx.html. 
En todo caso, si bien como indica González de Cossío, la salud arbitral de México "muestra groso modo un paciente sano", ${ }^{73}$ no es menos cierto que preocupa sobremanera la decisión de la Corte Suprema de ese país de limitar el principio Compétence-Compétence, como la recurrente utilización del amparo. ${ }^{74}$

En el caso del Perú, este país recientemente ha dictado una nueva Ley de Arbitraje (Decreto Legislativo No. 1071, publicado el 27 de junio de 2008 y vigente desde el $1^{\circ}$ de septiembre de 2008), que reemplaza a la Ley General de Arbitraje (Ley 26572) de 1996.

Si bien esta nueva Ley de Arbitraje identifica en su artículo 5 la existencia de arbitrajes internacionales, ${ }^{75}$ su normativa claramente estandarizada con la Ley Modelo de UNCITRAL (inclusive con los últimos cambios promovidos en el año 2006) aplica tanto a éstos, como a los arbitrajes domésticos o nacionales, ${ }^{76}$

73. Francisco González de Cossío, "Prognosis de la salud arbitral mexicana", ob. cit., p. 39.

74. Sobre este particular, leer a: Leonel Pereznieto Castro y James A. Graham, "El principio de la competencia arbitral en la actualidad mexicana". En: Lima Arbitration, Revista del Círculo Peruano de Arbitraje, No. 2, Lima, 2007,

www.limaarbitration.net/LAR2/leonel_pereznieto_castro_james_a_graham.pdf, pp. 240-249, Omar Guerrero Rodríguez y César Martínez Alemán, "Mexico: Judicial Precedents in Arbitration". En: Global Arbitration Review -The Arbitration Review of The Americas 2008, www.globalarbitrationreview.com/handbooks/4/sections/8/chapters/52/mexico; y, Francisco González de Cossío, "Prognosis de la salud arbitral mexicana", ob. cit., pp. 3-20.

75. El articulo 5 de esta ley dispone: "Artículo $5^{\circ}{ }^{-}$Arbitraje internacional.- 1. El arbitraje tendrá caracter internacional cuando en él concurra alguna de las siguientes circunstancias:

a. Si las partes en un convenio arbitral tienen, al momento de la celebración de ese convenio, sus domicilios en Estados diferentes.

b) Si el lugar del arbitraje, determinado en el convenio arbitral o con arreglo a éste, está situado fuera del Estado en que las partes tienen sus domicilios.

c) Si el lugar de cumplimiento de una parte sustancial de las obligaciones de la relación juridica o el lugar con el cual el objeto de la controversia tiene una relación más estrecha, está situado fuera del territorio nacional, tratándose de partes domiciliadas en el Perú.

2. Para efectos de lo dispuesto en el numeral anterior, si alguna de las partes tiene más de un domicilio, se estará al que guarde una relación más estrecha con el conventio arbitral".

De conformidad con este marco legal, necesariamente será internacional cualquier arbitraje que se desartolle entre personas 0 empresas domiciliadas en el extranjero, o cuando al menos una de las partes no domicilie en el Perú. La Ley General de Arbitraje se ubica en: www.amcham.org.pe/arbitraje/LEGISLACION/pdf/Ley_de_Arbitraje_Peru_2008.pdf.

76. En los hechos, la nueva Ley de Arbitraje es una ley monista, aunque a] arbitraje intenacional aplican algunas pocas disposiciones particulares: Artículo 2.2. (participación de Estados en arbitrajes internacionales); artículo 13.7 (ley aplicable al convenio arbitral); articujo 16.4 (excepción de convenio arbi- 


\section{posibilitando de esa manera la práctica plena del arbitraje den- tro de sus fronteras. ${ }^{77}$}

En el caso de Guatemala, si bien la Ley de Arbitraje 67-95 se aplica tanto al arbitraje nacional como al intemacional, ${ }^{78}$ a diferencia de la legislación mexicana contiene algunas pocas disposiciones locales, que en algunos casos pueden resultar poco amigables para la práctica del arbitraje internacional. ${ }^{79}$

tral); articulo 22.1 (no se requjere ser abogado para participar como árbitro en arbitrajes internacionales); artículo 57.2 (normas juridicas aplicables al fondo); $\mathrm{y}$, artículo 63.8 (posibilidad de renunciar al recurso de anulación). Sobre este particular, leer a: Fernando Cantuarias Salaverry y Roque J. Caivano, "La Nueva Ley de Arbitraje Pertana: Un nuevo salto a la modemidad", ob. cit., pp. 50-56.

77. El Tribunal Constitucional peruano, en sentencia de 28 de febrero de 2006, en el Expediente No. 6167-2005-PHC/TC LIMA FERNANDO CANTUARIAS SALAVERRY, ha establecido como Fundamento Jurídico Vinculante, lo siguiente: "I1. Es por tal motivo que este Tribunal conșidera conveniente reiterar la plena vigencia del principio de la 'kompetenz-kompetenz' previsto en el articulo $39^{\circ}$ de la Ley General de Arbitraje -Ley No. 26572-, que faculta a los árbitros a decidir acerca de las materias de su competencia... incluida las pretensiones vinculadas a la validez y eficacia del convenio. Este Colegiado resalta la suma importancia práctica que reviste dicho príncípio, a efectos de evitar que una de las partes, que no desea someterse al pacto de arbitraje, mediante un cuestionamiento de las decisiones arbitrales y/o la competencia de los árbitros sobre determinada controversia, pretenda convocar la participación de jueces ordinarios, mediante la interposición de cualquier acción de naturaleza civil y/o penal, y desplazar la disputa al terreno judicial". Este importante fallo se puede ubicar en: www.tc.gob.pe/jurisptudencia/2006/06167-2005.HC.html. White \& Case, International Disputes Quarterly -Focus on Aubitration in Latin America, Fall 2007, p. 18. "A decision by the Peruvian Constitutional Court reaffirsms Peru's commitment to arbitration as an effective means of dispute resolution, further consolidating a legal framework developed over the past two decades".

78. La Ley de Arbitraje 67-95 establece en su artículo 1: "La presente ley se aplicará al arbitraje nacional y al internacional, cuando el Jugar del arbitraje se encuentre en el territorio nacional...".

Artículo 2(1).- "Un arbitraje es internacional, cuando:

a) Las partes en un acuerdo de arbitraje tienen, al momento de su celebración, sus domicilios en estados diferentes, 0

b) Uno de los lugares siguientes está sinuado fuera del Estado en el que las partes tienen sus domicjlios:

i) El lugar del arbitraje, si este se ha determinado en el acuerdo de arbitraje o con arreglo al acuerdo de arbitraje.

ii) El lugar del cumplimiento de una parte sustancial de las obligaciones de la relación comercial o el lugar con el cual el objeto de litigio tenga una relación más estrecha; o

c) Las partes han convenido expresamente en que la cuestión objeto del acuerdo de arbitraje está relacionado con más đe un Estado",

79. Sobre el tema, leer a: Rafael Bemal Gutiérrez, "El arbitraje en Guatemala", Apoyo a la Justicia, Centro de Asbitraje y Conciliación (Cenac), Guatemala, 2000; y Marcos Ibargüen S., "Arbitration in Guatemala". En: The ICC International Court of Arbitration Bulletin -International Commercial Arbitration in Latin America. Special Sipplement, 1997, pp. 45-49. La ley se ubica en: www.servilex.com.pe/arbitraje/leyargu.html. 
Por último, Paraguay ${ }^{80}$ cuenta con una normativa bastante interesante basada en gran medida en la Ley Modelo de UNCITRAL. Sin embargo, llamamos la atención acerca de que esta ley permite en muchas oportunidades la intervención judicial, por lo que habrá que verificar la reacción de los tribunales de justicia locales para confirmar o descartar a Paraguay como un centro potencial de arbitrajes internacionales.

\section{II.4 Legislaciones que distinguen entre arbitrajes nacionales e internacionales y que han establecido Ios estándares idóneos para la práctica del arbitraje internacional dentro de sus fronteras, al haber adoptado la Ley Modelo de UNCITRAL}

En este grupo identificamos en primer lugar la Ley de Arbitraje Comercial Internacional 19971 de Chile, que permite contar con una buena legislación arbitral internacional, ${ }^{81}$ aunque

80. El artículo 3(c) de la Ley $1879 / 02$ de Arbitraje y Mediación considera como arbitraje internacional "aquel en el cual:

1. las partes de un acuerdo de arbitraje tienen, al momento de la celebración de ese acuerdo, sus establecimientos en estados diferentes; 0

2. el lugar del cumplimiento de una parte sustancial de las obligaciones de la relación comercial o el lugar con el cual el objeto de likigio tenga una relación más estrecha, esté situado fuera del Estado en el que las partes tienen sus establecimientos". La ley se ubica en:

www.servilex.com.pe/arbitraje/paraguay/ley.doc.

81. El articulo I de esta ley publicada el 30 de setiembre de 2004 dispone: "Articulo 1.- Ámbito de aplicación.

1) Esta ley se aplicará al arbitraje comercial internacional, sin perjuicio de cualquier tratado multilateral o bilateral vigente en Chile.

2) Las disposiciones de esta ley, con excepción de los artículos 8, 9, 35 y 36, se aplicarán únicamente si el lugar del arbitraje se encuentra en el territorio nacional.

3) Un arbitraje es internacional si;

a) Las partes en un acuerdo de arbitraje tienen, al momento de la celebración de ese acuerdo, sus establecimientos en Estados diferentes; 0

b) Uno de los lugares siguientes está siłuado fuera del Estado en el que las partes tienen sus establecimientos:

i) El lugar del arbitraje, si este se ha determinado en el acuerdo de arbitraje o con arreglo al acuerdo de arbitraje;

ii) El lugar del cumplimiento de una parte sustancial de las obligaciones de la relación comercial o el lugar con el cual el objeto del litigio tenga una relación más estrecha; 0

c) Las partes han convenido expresamente en que la cuestión objeto del acuerdo de arbitraje está relacionada con thás de un Estado. 


\section{mantiene vigente un marco legal aplicable al arbitraje nacional o doméstico que es ineficiente y anticuado.}

Aquí pues será imprescindible confirmar que la controversia será considerada en este país como "comercial internacional", ya que, de lo contrario, y como ya indicamos, se aplicará una regulación anticuada e ineficiente. ${ }^{82}$

Por último, en Bermuda puede decirse que se presenta una situación similar a la de Chile, ya que cuenta con una excelente Ley de Conciliación y Arbitraje Internacional (1993), ${ }^{83}$ adoptada

4) A los efectos del numeral 3) de este artículo:

a) $\mathrm{Si}$ alguna de las partes tiene más de un establecimiento, el establecimiento será el que guarde una relación más estrecha con el acuerdo de arbitraje.

b) Si una parte no tiene ningún establecimiento, se tomará en cuenta su residencia habitual.

5) Esta ley no afectará a ninguna otra ley en virtud de la cual determinadas controversias no sean susceptibles de arbitraje o se puedan someter a arbitraje úticamente de conformidad con disposiciones que no sean las de la presente ley". La ley se ubica en: www.servilex.com.pe/arbitraje/chile/leyarbinter.html. Sobre el particular, leer a: Cristián Conejero Roos, "The New Chilean Arbitration Law and the Influence of the Model Law". En: Journal of International Arbitration, vol. 22, nro. 2, 2005, pp. $149-162$.

82. La legislación preexistente a la Ley sobre Arbitraje Comercial Internacional mantiene su vigencia para el arbitraje local, estableciéndose asi un régimen dual: un arbitraje internacional moderno, frente a un arbitraje doméstico o nacional ineficiente y anticuado. Sobre la legislación arbitral chilena previa a la reforma, que mantiene su vigencia para los arbitrajes nacionales o domésticos, Carlos Urenda Z., "Recent Developments in National and International Arbitration in Chile", documento presentado en el First Annual Miami International Arbitration Conference, ICDR International Centre for Dispute Resolution y Stell Hector Davis Intemational, Miami, 2003, p. 3, afirma que "[l]a aplicación del derecho interno es manifiestamente insuficiente para hacer atractivo someter a árbitros chilenos 0 a procedimientos locales inapropiados los arbitrajes internacionales y atraer la confianza de las grandes empresas de todos los países del mundo que celebran toda clase de contratos cuando deciđen hacer negocios en Chile". Sobre el tema, leer a: Hernán G. Somerville, "Arbitration in Chile". En: The ICC Intemational Court of Arbitration Bulletin -International Commercial Arbitration in Latin America, Special Supplement, 1997, pp. 15-20; y Carlos Eugenio Jorquiera y Karin Helmlinger, "Chile". En: International Arbitration in Latin America, Nigel Blackaby, David Lindsey y Alessandro Spinillo (editores), Kluwer Law International, La Haya, 2002, pp. 89-110. La ley aplicable al arbitraje doméstico o nacional se ubica en: www.servilex.com.pe/arbitraje/chile/cpccl.html.

Este sistema legal dual que propone normas legales diametralmente diferentes parece que está generando problemas en este país. En efecto, nosotros hemos tenido acceso a dos fallos judiciales (de la Cuarta Sala de la Corte de Apelaciones de 3 de mayo de 2006 y de la Primera Sala de la Corte de Apelaciones de 25 de mayo de 2006), en los que si bien se denegaron los recursos interpuestos, lo cierto es que en ambos casos la discusión gitó en torno a determinar si los arbittajes iniciados eran nacionales o internacionales, ya que de ello dependia la aplicación de la normatividad correspondiente. Obviamente preocupa que deba recurrirse al Poder Judicial previo al inicio de un arbitraje para que se determine si se aplica la moderna Ley sobre Arbitraje Comercial Internacional o la vetusta legislación doméstica de arbitraje.

83. La Ley de Conciliación y Arbitraje Internacional se ubica en: www.kluwerarbitration.com/arbitration/art/home/ipn/default.asp?ipn $=11868$. 
conforme a la Ley Modelo de UNCITRAL y, al mismo tiempo, mantiene una menos eficiente Ley de Arbitraje de 1986, aplicable a los arbitrajes domésticos. ${ }^{84}$

\section{Consecuencias y Alternativas de Solución}

Como hemos identificado, en realidad son muy pocas las legislaciones arbitrales que pueden ser consideradas como modernas y amigables para la práctica del arbitraje internacional en la región. ${ }^{85}$

Con este estado de cosas, resulta muy difícil fomentar el intercambio comercial privado entre nuestras fronteras, ya que las partes se ven imposibilitadas, o al menos seriamente limitadas para poder pactar el arbitraje, simplemente porque la mayoría de nuestros países no están preparados para acoger arbitrajes comerciales internacionales.

En efecto, hoy por ejemplo es muy difícil que una empresa peruana y otra colombiana pacten el arbitraje (salvo que quieran y puedan hacerlo en México, Estados Unidos o Europa) en algu-

84. Narinder K. Hargun y Jeffrey P. Elkinson, "Bermuda". En: International Handbook on Commercial Arbitration, J. Paulsson (editor), Supplement 18, 1994, p. 1. "The Bermuda International Conciliation and Arbitration Act 1993 .. became operative on 29 June 1993. The 1993 Act provides that the UNCITRAL Model Law on International Commercial Arbitration... shall have the force of law in Bermuda... Bermuda has two systems of arbitration law; the Model Law, applying to international arbitrations... and the Arbitration Act 1986... applying to domestic arbitration".

85. Centro de Arbitraje y Mediación de la Cámara de Comercio de Santiago, "Ley de Arbitraje Comercial Internacional para Chile". En:

www.camsantiago.com/actividades/noticias/ley_arb_com_internac.html, p. 3. "El último libro sobre arbitraje internacional en Latinoamérica de los autores Nigel Blackaby y otros, editado el año 2002 por Kluwer Law International (International Arbitration in Latin America)... [identifica] que en los arbitrajes comerciales internacionales que tienen contrapartes latinoamericanas, se escogen hoy como sedes en forma habitual Nueva York, París y Miami. Madrid no es escogido como sede, aungue debiera serlo por razones de idioma, por la falta de una legislación adecuada [sin embargo, España acaba de aprobar una excelente Ley de Arbitraje]. En el caso de partes de babla portuguesa, como ocurre con Brasil, to normal sería que se escoja a Lisboa como sede, cuestión que tampoco ocurre por las mismas razones de Madrid y por haber suscrito además solo en forma miry reciente la Convención de Nueva York. Si alguna de las partes insiste en designar como sede a un país de la Región, el escogido por excelencia resulta ser México. Otros posibles elegidos, aunque con dificultades por razones de estabilidad política y económica, son Perú y Colombia. Chile [que ahora cuenta con una excelente Ley de Arbitraje Internacional] y Argentina no son escogidos bajo ningun respecto, por no contar con una ley de arbitraje internacional".

336 Revista Ecuatoriana de Arbitraje 
no de los Estados que conforman la Comunidad Andina de Naciones, simplemente porque Bolivia, Colombia y Ecuador cuentan, para estos efectos, con legislaciones arbitrales poco amigables. Además, en este ejemplo, el Perú no siempre es una alternativa, debido al tema de la neutralidad.

Pero, además, aun cuando se pudiera afirmar que la gran mayoría de los Estados latinoamericanos cuentan con legislaciones aptas para la práctica del arbitraje comercial internacional (que como hemos identificado no es el caso), igual tendríamos que enfrentar un significativo problema adicional:

"La existencia de distintos ordenamientos jurídicos internos, con variados requisitos para la validez y eficacia del arbitraje, genera inconvenientes de tal magnitud que pueden derivar en la inoperatividad del arbitraje internacional, pues constituye un hecho innegable que las naciones legislan con el objeto de regular su realidad interna, y el arbitraje internacional excede largamente ese ámbito". 86

En otras palabras, aun cuando la gran mayoría de los países latinoamericanos estuvieran preparados para recibir arbitrajes comerciales internacionales dentro de sus fronteras, lo cierto es que muchos de ellos cuentan con tantas disposiciones locales o particulares, que resulta una tarea casi titánica el poder preverlas al momento de pactar el arbitraje. ${ }^{87}$

Ante este estado de cosas, la primera alternativa de solución sería intentar que todos los países de la región unificaran sus legislaciones arbitrales.

86. Felipe Osterling Parodi, "La necesidad de unificar las normas sobre arbitraje en América Latina como consecuencia de la globalización". En: Revista lberoamericana de Arbitraje, www.servilex.com.pe/arbitraje/peru/artfope.html, p. 2. Gabrielle Kaufmann-Kohler, "Intemational Commercial Arbitration: Globalization of Arbitral Procedure". En: Vanderbilt Journal of Transnational Law, vol. 36, 2003, p. 1322. "Consensus on principles does not mean agreement on details".

87. Sobre este particular, recomendamos leer a: Cristian Conejero Roos, "Análisis comparativo de la influencia de la Ley Modelo de la CNUDMI en Latinoamérica". En: Revista Internacional de Arbitraje, nro. 4, Bogotá, 2006, pp. 13-103. 
Si bien expertos de la talla de Caivano consideran viable esta posibilidad, ${ }^{88}$ nosotros no creemos que nos llevará a buen puerto.

La razón es muy simple: Nadie niega que, en poco más de diez años, Latinoamérica ha dado señales claras en favor del arbitraje ${ }^{89}$ y que, como explica Griguera, ${ }^{90}$ las nuevas legislaciones arbitrales están orientadas a la adopción de principios y reglas sobre arbitraje comercial que prevalecen en el mundo y que cuentan con un amplio consenso. ${ }^{91}$

\section{Sin embargo, aquí el problema se presenta con las pequeñas} ("grandes") diferencias, con las "excepciones" localistas que impiden una clara armonización, y, en muchos casos, con la existencia de importantes vacíos. ${ }^{92}$

88. Roque J. Caivano, "El arbitraje y los procesos de integración (su futuro en el Mercosur)", ob. cit, p. 783. "La generalizada ratificación de las convenciones sobre reconocimiento y ejecución de laudos arbitrales extranjeros (Nueva York 1958 y Panamá 1975) y los procesos de unificación de las legislaciones internas sobre arbitraje va logrando superar los escollos que antiguamente hacían de los laudos arbituales internacionales una herramienta poco eficaz frente a la resistencia de los jueces nacionales a darles cumplimiento forzado". Ulises Pitti $G$., "La regulación del arbitraje, la conciliación y la mediación en la legislación panamena", ob. cit., p. 2. "[L]os paises deben realizar reformas tendientes a la modernización económica y jurisdiccional, antes de ingresar a las zonas de integración. Ello hace imperativo crear y desarrollar nuevas leyes para la solución expedita de las controversias, y la ratificación y la ejecución de las Convenciones de Panamá de 1975 y de Nueva York de 1958, dentro del ámbito de la aplicación de los Tratados de integración".

89. Rubén Santos Belandro, "Una nueva actitud de los Estados latinoamericanos hacia el arbitraje". En: www.servilex.com.pe/arbitraje/colaboraciones/congreso_internacional_rsb.html, p. 6. "La consecuencia de este enorme esfuerzo codificador es que al dia de hoy todas las legislaciones nacionales del continente -en mayor o menor medida-apoyan y alientan al arbitraje".

90. Horacio A. Griguera Naón, "Recent Trends Regarding Commercial Arbitration in Latin America", ob. cit., p. 96. "[T] he new texts do not denote the creation of a 'regional' arbitration culture that would differ from general trends fashioning the development of commercial arbitration in the world. On the contrary, the new legislative trends in Latin Ametica are oriented towards the adoption of the general principles and rules concerning commercial arbitration prevailingly accepted or enjoying general consensus in the milieu of arbitral experts and practitioners, primarily as enshrined in the Model Law".

91. Cristian Conejero Roos, "Análisis comparativo de la influencia de la Ley Modelo de la CNUDMI en Latinoamérica". En: Revista Internacional de Arbitraje, nro. 4, Bogotá, 2006, pp. 31-33.

92. Nigel Blackaby, David M. Lindsey y Alessandro Spinillo, "Overview of Regional Development", ob. cit., p. 7. "Each exotic or unusual element is a good reason for foreign investors not to accept the location of the arbitration in the country in question. The desire of legislators to demonstrate that their country is different can only be lamented since the success of intemational arbitration in developing countries depends on the adoption of common solutions". Nigel Blackaby y Sylvia Noury, "International Arbitration in Latin America", ob. cit., p. 2. "Unfortunately, several [Latin American] countries did not opt for the Model Law when updating theit arbitration laws, insisting instead on developing their own projects. They took certain elements of the Madel Law but introduced aspects of their own historic arbitral procedures which they considered appropriate or which raised issues of public policy, without examining the effect of these elements on foreign parties... Each exotic or unu- 
Creemos, por tanto, que lo que se requiere es la adopción de convenios internacionales que permitan corregir el actual estado de cosas.

Para estos efectos, podría pensarse en la adopción de textos como los Protocolos Nos $3 / 98$ y 4/98 que contienen una normativa específica en el ámbito del arbitraje comercial internacional privado, y que actualmente resultan aplicables a los cuatro países firmantes del Mercosur y entre éstos y los dos miembros que no son plenos (Bolivia y Chile), respectivamente. ${ }^{93}$

Se trata de dos instrumentos internacionales (aunque sustancialmente similares) de la mayor importancia para la consolidación del arbitraje comercial internacional en Latinoamérica, ya que, como explica Fraser, ${ }^{94}$ no solo regulan el reconocimiento y la ejecución de los laudos arbitrales foráneos, sino que, además, y principalmente, establecen una regulación completa del arbitraje comercial internacional hacia el interior de los países que conforman el Mercosur y entre estos y sus socios (Chile y Bolivia).

sual element is a good reason for foreign investors not to accept the seat of the arbitration in the country in question. The desire of legislators to demonstrate that their country is different can only be lamented since the success of international arbitration in developing countries depends on the adoption of common solutions".

93. Alicia M. Perugini Zanetti, "Arbitraje comercial internacional en el Mercosur". En: Avances del Derecho Internacional Privado en América Latina. Jan Kleinheisterkamp y Gonzalo A. Lorenzo Idiarte (coordinadores), Fundación de Cultura Universitaria, 2002, pp. 636-637. "Tales circunstancias normativas alentaron a la Reunión de Ministros de Justícia a instruir a la Comisión Técnica para que elabore un texto común que responda a los problemas insatisfechos y que pernita un solo lenguaje arbitral en los Estados Partes del MERCOSUR, Bolivia y Chile. Una solución unívoca tornaría previsible para los protagonistas del proceso arbitral -las partes, los árbitros y los jueces-el tratamiento que recibirá este sistema alternativo adversial en las distintas etapas del proceso en cada uno de los Estados. Por otra parte, un texto común tendría un efecto residual: contribuiría por sí mismo a promover el arbitraje, lamentablemente, no demasiado difundido en la práctica de los diferentes países đe la región".

94. David Fraser, "Arbitration in Latin America: An Overview". En: International Arbitration Law Review, vol. 5, nro. 2, 2002, p. 65. "This is not a convention dealing only with the recognition and enforcement of arbitration awards in the Mercosur countries; it is a complete code of arbitration law, including enforcement of foreign awards, regulating arbitration of international commercial contracts. It has been described as "a new regime". 
En efecto, si bien no pretendemos analizar a fondo los protocolos, ${ }^{95}$ destacamos el importante esfuerzo realizado por intentar uniformizar el tratamiento de los arbitrajes comerciales internacionales, ${ }^{96}$ abordando para el efecto todas las áreas del arbitraje, como son: el ámbito de aplicación, el convenio arbitral (que se le da el nombre de convención arbitral), el procedimiento arbitral, el tribunal arbitral, la competencia del tribunal arbitral, el laudo arbitral, los recursos contra los laudos arbitrales y el reconocimiento y la ejecución de los laudos arbitrales extranjeros. ${ }^{97}$

Sin embargo, al mismo tiempo, identificamos algunos graves errores, como son: se otorga demasiada deferencia a la legislación del lugar del arbitraje para la regulación de algunos temas sensibles e importantes; su ámbito de aplicación es complicado y poco claro $^{98} \mathrm{y}$, al mismo tiempo, limitado ${ }^{99}$; existe una equívoca referencia a tratados distintos a la Convención de Nueva York para el reconocimiento y la ejecución de laudos arbitrales extranjeros; ${ }^{100}$ y se establecen ciertas condiciones y requisitos extraños a los estándares internacionales. 101

95. Los protocolos se ubican en: www.sice.oas.org/trade/mresrs/decisions/DEC0398.asp y www.sice. oas.org/trade/muresrs/decisions/DEC0498.asp.

96. David M. Lindsey y Alessandro Spinillo, "Intemational Commercial Arbitration in Mercosur". En: International Arbitration in Latin America, Nigel Blackaby, David Lindsey y Alessandro Spinillo (editores), Kluwer Law International, La Haya, 2002, p. 259, identifican que ambos protocolos han adoptado, aunque solo en parte, la Ley Modelo de UNCITRAL.

97. Luis O. Andomo, "El Acuerdo sobre Arbitraje Comercial Internacional del Mercosur. Su posibilidad de aplicación a las empresas actuantes en el contrato de transporte multimodal de mercaderias". En: Revista Juridica Virtual del Programa de Investigación sobre Armonización de las Legistaciones, www.salvador.edu.ar/sv91mul l-pub01-1-1-03.htm. Para un análisis exhaustivo, leer a: Alicia $\mathrm{M}$. Perugini Zanetti, "Arbitraje comercial internacional en el Mercosur", ob. cit., pp. 638-666.

98. Nigel Blackaby, David M. Lindsey y Alessandro Spinillo, "Overview of Regional Development", ob. cit., p. 8. "[T]he scope of application of the agreements is complicated and unclear". David $\mathbf{M}$. Lindsey y Alessandro Spinillo, "International Commercial Arbitration in Mercosut", ob. cit., p. 279. "The MAA are an attempt to improve arbitration legislation in the region, but they present important flaws in key matters such as their scope of application...".

99. Maria Rosa Cattaneo, "El arbitraje comercial internacional en el Mercosur, Bolivia y Chile. Su desartollo reciente y perspectivas futuras". En: Revista Iberoamericana de Arbitraje, www.servilex.com.pe/arbitraje/congresopanama/b-10.html, p. 5. "El ámbito de aplicación material es, a mi criterio, muy reducido, por cuanto se restringe a los contratos comerciales entre particulares, personas fisicas o jurídicas del derecho privado... quedando excluidos otros negocios comerciales".

100. Jonathan Van Ee, "MERCOSUR Arbitration: A New Regime". En: International Arbitration Law Review, vol. 4, nro. 2, 2001, p. 58. “Article 23 of the Mercosur Agreement provides that enforcement of foreign arbitral awards in Mercosur countries shall be governed by a Metcosur Protocol, the 
Aun así, como ya hemos expresado, ambos protocolos son valiosos en la tarea de trabajar por la consolidación de la práctica del arbitraje comercial internacional en Latinoamérica, por lo que deberían de servir de base para una acción conjunta más allá del MERCOSUR. ${ }^{102}$

En realidad, si logramos establecer reglas uniformes que habiliten a nuestros comerciantes y empresarios pactar el arbitraje en cualquiera de nuestros países, permitiendo así que elijan el lugar del arbitraje exclusivamente en razón de criterios como la neutralidad, los costos y los servicios, habremos avanzado en la dirección correcta para promover un verdadero crecimiento del comercio en nuestra región. ${ }^{103}$

Interamerican Convention, and the Montevideo Convention. Notably absent is the New York Convention, which is significant because, as the most comprehensive arbitration regime, one could expect to find incorporated in some manner. This absence is the biggest hurdle to incorporating the New York Convention through the Mercosur Agreement". Horacio A. Griguera Naón, "Recent Trends Regarding Commercial Arbitration in Latin America", ob. cit, p. 105. "[O]ne wonders why Mercosur countries did not follow the easier path of just ratifying the Panama or New York Conventions instead of creating and adopting special compacts governing the recognition and enforcement of atbitral awards for the Mercosur region".

101. Jonathan Van Ee, "MERCOSUR Arbitration: A New Regime", ob. cit., p. 57. "[T]he Mercosur Agreement uses distinctions the foreign lawyer may not be familiar with such as an arbitration agreement's 'formal validity' and 'intrinsic validity', which are both different from issues regarding the merits of the dispute. Also, there is the requirement that arbitrators abide by principles of contradictory (contradictorio) and free persuasion (libre convencimiento). If these distinctions are not respected an award may be set aside".

102. Horacio A. Griguera Naón, "Recent Trends Regarding Commercjal Arbitration in Latin America", ob. cit., p. 99, entiende lo mismo cuando afima: "These coordinated efforts, when properly undertaken from a common multinational approach, should have the beneficial effect of unifying rules and criteria for dealing with issues regarding arbitration procedures, arbitral clauses and awards in the different Latin American countries and hopefully thus enhance the predictability of the corresponding solutions irrespective of the national jurisdiction called to decide on them".

103. Jan Kleinheisterkamp, "Conflict of Treaties on International Arbitration in the Southern Cone", ob. cit., p. 688. "Only if business could prosecute its claims beyond the national borders and without the traditional obstacles to recognition and enforcement, the risks of engaging with the interweavement promoted by the politics of integration could become manageable, thus breaking the path to the promised synergetic effects". 